\title{
Mitogen-Activated Protein Kinase 6 and Ethylene and Auxin Signaling Pathways Are Involved in Arabidopsis Root-System Architecture Alterations by Trichoderma atroviride
}

\author{
Hexon Angel Contreras-Cornejo,, Jesús Salvador López-Bucio, ${ }^{2}$ Alejandro Méndez-Bravo, ${ }^{1}$ \\ Lourdes Macías-Rodríguez, ${ }^{1}$ Maricela Ramos-Vega, ${ }^{2}$ Ángel Arturo Guevara-García, ${ }^{2}$ and \\ José López-Bucio'1 \\ ${ }^{1}$ Instituto de Investigaciones Químico-Biológicas, Universidad Michoacana de San Nicolás de Hidalgo. Edificio B3, Ciudad \\ Universitaria. C. P. 58030, Morelia, Michoacán, México; ${ }^{2}$ nstituto de Biotecnología, Universidad Nacional Autónoma de México, \\ Apartado Postal 510-3, 62250 Cuernavaca, Morelos, México
}

Submitted 6 January 2015. Accepted 30 January 2015.

\begin{abstract}
Trichoderma atroviride is a symbiotic fungus that interacts with roots and stimulates plant growth and defense. Here, we show that Arabidopsis seedlings cocultivated with $T$. atroviride have an altered root architecture and greater biomass compared with axenically grown seedlings. These effects correlate with increased activity of mitogen-activated protein kinase 6 (MPK6). The primary roots of mpk6 mutants showed an enhanced growth inhibition by $T$. atroviride when compared with wildtype (WT) plants, while T. atroviride increases MPK6 activity in WT roots. It was also found that $T$. atroviride produces ethylene (ET), which increases with L-methionine supply to the fungal growth medium. Analysis of growth and development of WT seedlings and etr1, ein2, and ein3 ET-related Arabidopsis mutants indicates a role for $\mathrm{ET}$ in root responses to the fungus, since etr1 and ein2 mutants show defective root-hair induction and enhanced primary-root growth inhibition when cocultivated with $T$. atroviride. Increased MPK6 activity was evidenced in roots of ctrl mutants, which correlated with repression of primary root growth, thus connecting MPK6 signaling with an ET response pathway. Auxin-inducible gene expression analysis using the DR5:uidA reporter construct further revealed that ET affects auxin signaling through the central regulator CTR1 and that fungal-derived compounds, such as indole-3-acetic acid and indole-3-acetaldehyde, induce MPK6 activity. Our results suggest that $T$. atroviride likely alters root-system architecture modulating MPK6 activity and ET and auxin action.
\end{abstract}

Plants integrate a network of external stimuli, including light, temperature, nutrients, soil $\mathrm{pH}$, and the presence of organisms such as other plants, microbes, nematodes, and insects to adjust their growth and development. Plant developmental patterns are regulated by different phytohormones, mainly auxins, cytokinins and ethylene (ET), while interactions among hormonal

Corresponding author: José López-Bucio; Telephone: +52 (443) 3265788; Fax: +52 (443) 3265788; E-mail: jbucio@umich.mx

*The $\boldsymbol{e}$-Xtra logo stands for "electronic extra" and indicates that five supplementary figures are published online.

(c) 2015 The American Phytopathological Society pathways modulate morphogenesis (Acharya and Assmann 2009).

The rhizosphere is the soil region that is influenced by roots due to exudation of carbohydrates, amino acids, organic acids, and mucilage, which represent a rich source for nutrients for microbial populations. On the other hand, microorganisms inhabiting roots may have a strong influence on plant growth and health. Root-microbe communication occurs through release of diverse signaling molecules, leading to symbiosis events that improve plant fitness directly or indirectly. As an example, most angiosperms develop symbiotic interactions with fungal species, such as mycorrhizal fungi, Piriformospora indica, and Trichoderma spp., which may act in biocontrol of pathogens, activating defense responses, boosting nutrient uptake, or producing growth-regulating substances (ContrerasCornejo et al. 2013; Khatabi et al. 2012).

Trichoderma species are frequently found in agricultural soils as common inhabitants of the rhizosphere. T. virens and T. atroviride produce auxins and auxin-like compounds that affect both root-hair and lateral-root development (ContrerasCornejo et al. 2009, 2014). Root responses to auxin are mediated by three protein families, i.e., the AUXIN RESPONSE FACTOR (ARF) family of transcription factors, responsible for the regulation of auxin-inducible gene expression, the AUXIN/ INDOLE-3-ACETIC ACID (AUX/IAA) transcriptional inhibitors, which interact with ARF to prevent their action, and F-box proteins that are part of the ubiquitin protein ligase $\mathrm{SCF}^{\mathrm{TIR} 1}$ complex controlling the rapid ubiquitin-mediated degradation of AUX/IAA proteins in response to auxin (Růzicka et al. 2007). Inside cells, auxin binds to the TRANSPORT INHIBITOR RESPONSE 1/AUXIN SIGNALING F-BOX (TIR1/AFB) family of F-box proteins, which are subunits of the SCF E3-ligase protein complex, to lead the ubiquitination and the proteasome-mediated specific degradation of AUX/IAA transcriptional repressors. Subsequently, the ARF are released to activate auxin-inducible gene expression (Dharmasiri et al. 2005).

In a recent report, the potential of $T$. virens and $T$. atroviride to confer salt tolerance to Arabidopsis seedlings through an auxin signaling pathway was explored. It was found that salt represses plant growth and root development in a dosedependent manner blocking auxin signaling. Analysis of WT and ETHYLENE INSENSITIVE ROOT 1 (eirl), auxl-7, 
arf7arf19, and tirlafb2afb3 auxin-related mutants, revealed a key role for IAA signaling in mediating salt tolerance. Interestingly, T. virens (Tv29.8) and T. atroviride (IMI 206040) promoted plant growth in both normal and saline conditions that was related to the induction of lateral roots and root hairs through auxin signaling (Contreras-Cornejo et al. 2014).

ET also regulates root developmental processes, acting either synergistically or independently of auxin signaling (Muday et al. 2012). For instance, ET promotes root-hair initiation and elongation but, in contrast to auxin, ET inhibits lateral root formation and elongation (Bleecker and Kende 2000; Johnson and Ecker 1998; Pitts et al. 1998). ET is perceived by ETbinding receptors, such as ETHYLENE RESPONSE 1 (ETR1) and ETHYLENE RECEPTOR SUBFAMILY 1 (ERS1). The activation of these receptors causes repression of CONSTITUTIVE TRIPLE RESPONSE 1 (CTR1), which, in turn, permits ETHYLENE INSENSITIVE 2 (EIN2) to relay the ET signal to the transcription factors EIN3 and ETHYLENE-INSENSITIVE3LIKE (EIL). EIN3 activates ETHYLENE RESPONSE FACTOR 1 (ERF1), inducing the expression of ET-responsive genes (Huang et al. 2003; Kieber et al. 1993; Sánchez-Rodríguez et al. 2010). Loss-of-function mutations in ETHYLENE-INSENSITIVE 2 and 3 severely limit the plant's response to ET (Chao et al. 1997).

Intracellular signaling processes that translate microbial perception into genetic, metabolic, and developmental responses in roots are the focus of current plant-microbe interactions research. Important signaling pathways involved in recognition of microbial elicitors, such as pathogen- or microbe-associated molecular patterns, as well as growth regulating substances such as auxins or ET or both include mitogen-activated protein kinase (MAPK) cascades (Zhang et al. 2006). MAPKs act through phosphorylation events in which a MAPK kinase kinase (MPKKK) phosphorylates a MAPK kinase and this, in turn, activates a MAPK (MPK6) to translate external stimuli into cellular responses (Colcombet and Hirt 2008). MAPKs mediate responses to a variety of signaling molecules, such as reactive oxygen species, flagellin, UV light, brassinosteroids, ET, and nitric oxide. Indeed, MAPKs phosphorylate different substrates, including other kinases, phosphatases, or transcription factors, to regulate stomatal, embryo, and root development (Brader et al. 2007; Yoo et al. 2008). In a recent study, the role of MPK6 in Arabidopsis embryo development and in postembryonic root system architecture was unraveled (López-Bucio et al. 2014). The mpk6 mutation caused alterations in embryo development and in root architecture characterized by a primary root longer than that of the WT, accompanied by significantly increased lateral root initiation and more and longer root hairs. Apparently, the increment in primary root growth resulted from an enhanced cell production and cell elongation. These data demonstrated that MPK6 acts as a repressor of primary and lateral root development as well as root-hair differentiation and growth (López-Bucio et al. 2014).

So far, all described information indicates that Trichoderma spp. improve plant growth and modulate root architecture by producing auxins and other signaling molecules, while the mechanisms by which the plant senses the fungal signals remain to be characterized. In this work, we show that T. atroviride regulates root architecture and increases MPK6 activity, likely depending on ET and auxin signaling. It was found that ET, IAA, and IAAld (indole-3-acetaldehyde) produced by the fungus induce MPK6 activity. Also, molecular and phenotypical analysis of ET-related mutants ctr1, ein2, ein3, and etrl indicate an important role of ET in T. atroviride modulation of root architecture. Further analysis of auxinrelated gene expression in WT seedlings and ctrl mutants expressing the DR5:uidA construct showed that the effect of ET on root growth and differentiation is apparently mediated by auxin signaling, thus indicating that an auxin-ET crosstalk involving MPK6 fine-tunes seedling growth and development in response to a beneficial soil fungus.

\section{RESULTS}

\section{T. atroviride regulates root architecture and increases MPK6 activity.}

The effects of T. atroviride on Arabidopsis growth and development were investigated using an in vitro fungus-plant interaction system. To test the possibility that some volatile or diffusible fungal compounds could be involved in promoting plant growth, fungal spores were placed at a distance of $5 \mathrm{~cm}$ from the primary root tip. In such experimental conditions, without any root-mycelium physical contact, T. atroviride clearly promoted lateral-root and root-hair formation and increased total plant biomass production (Supplementary Fig. S1).

MPK6 regulates plant responses during plant-microbe interactions (Merkouropoulos et al. 2008; Ren et al. 2008), and recently, it was considered as a negative regulator of root development, as the mpk6 mutant produced more lateral roots and root hairs than WT seedlings (López-Bucio et al. 2014). Here, the possible involvement of MPK6 in root responses to T. atroviride was investigated. In experiments in which WT (Col-0) and mpk6 mutant seedlings were grown side by side on axenic agar-solidified Murashige and Skoog (MS) $0.2 \times$ medium and in the same conditions but in the presence of the fungus, root-hair parameters were analyzed 2 days after inoculation (d.a.i.). It was found that T. atroviride increased $50 \%$ the length of root hairs and $25 \%$ the root-hair density in WT plants (Fig. 1A to F). Meanwhile, when grown under axenic conditions, mpk6 mutants produced significantly longer root hairs and increased root-hair density than WT seedlings, but when cocultivated with $T$. atroviride, the increase in the length of root hairs and root-hair density was similar to the WT (Fig. $1 \mathrm{~A}$ to $\mathrm{F})$.

In contrast to root hairs, the primary roots of inoculated $m p k 6$ plants showed an enhanced growth inhibition by $T$. atroviride when compared with WT plants, whose primary root growth was unaffected by the presence of the fungus (Fig. 2A). In addition, in relation to their respective axenic controls, the lateral root number of WT plants cocultivated with $T$. atroviride increased $400 \%$, while inoculated $m p k 6$ plants increased only $40 \%$ (Fig. 2B). The greater ability of mpk6 mutants to develop lateral root meristems under axenic conditions may explain why $T$. atroviride did not increase further the number of lateral roots in the mutant as compared with the WT.

To test if T. atroviride has some effect on MPK6 activity, an in-gel MAPK assay was performed, using fungus-challenged plant material from Arabidopsis WT seedlings in which no contact between the mycelium and the primary root had been established (4 d.a.i.) and another in which $30 \%$ of the root system was covered by the fungal colony ( 6 d.a.i.). In both cases, we found that MPK6 activity increases after inoculation (Fig. 2C). The induction of MPK6 activity by $T$. atroviride was absent in mpk6 Arabidopsis mutants (Supplementary Fig. S2). These results show that $T$. atroviride modulates plant growth and that MPK6 probably is involved in this signaling pathway.

\section{T. atroviride releases $\mathrm{ET}$ in response to L-methionine supply.}

Several rhizospheric fungi are able to produce plant hormones (Barbieri and Galli 1993; Contreras-Cornejo et al. 2009; Malhotra and Srivastava 2008; Patten and Glick 2002; Splivallo 
et al. 2009; Suzuki et al. 2003). A major effect of T. atroviride is regulating root-hair morphogenesis, a trait that is under ET and auxin control, opening the possibility that the fungus could produce this kind of compound. As we already identified IAA and auxin-related compounds produced by Trichoderma spp. (Contreras-Cornejo et al. 2009), we now conducted experiments aimed at evaluating whether these compounds were present in the growth medium after growth of $T$. atroviride colonies by gas chromatography (GC)-mass spectrometry. All three compounds, namely IAA, IAAld, and ICAld (indole-3carboxaldehyde), could be identified as some of the metabolites produced by $T$. atroviride (Supplementary Fig. S3). ET production by $T$. atroviride was also determined by GC-mass spectrometry analyses. For this, the fungus was grown on petri dishes and the volatile compound was released to the headspace of the plates. We identified ET (retention time: $2.39 \mathrm{~min}$ ) released by the fungal colony at 4 and 6 days of growth (Fig. 3A and B). Importantly, when $1 \mathrm{mM} \mathrm{L-methionine} \mathrm{was} \mathrm{added} \mathrm{to} \mathrm{the}$ culture medium, the production of ET was substantially increased (Fig. 3B). These data confirm that $T$. atroviride produces auxins and show that it could induce root architectural changes by producing ET.

\section{T. atroviride modulates root-hair development through ET signaling.}

ET regulates root-hair elongation through binding to the ET receptor ETR1, which regulates CTR1, EIN2, and EIN3 downstream (Pitts et al. 1998; Yoo et al. 2008). To determine whether T. atroviride induced root-hair formation through ET signaling, we analyzed the effects of fungal cocultivation on root-hair length and density in WT and etr 1-1, ein2-1, and ein3-1 ET-related mutant lines. As previously reported (Pitts et al. 1998), axenically grown etr $1-1$ and ein2-1 mutants showed a significant reduction in root-hair length and density when compared with WT plants, whereas the ein3-1 mutant showed only a small but yet significant reduction (Fig. 4A to J). In these experiments, T. atroviride clearly increased root-hair length and density in the WT, an effect that was absent in the three ET-related mutants analyzed (Fig. 4A to J). Treatment with silver nitrate $\left(\mathrm{AgNO}_{3}\right)$, an ET inhibitor, impaired root-hair development in WT and mpk6 mutants grown under axenic conditions or cocultivated with T. atroviride. In contrast, treatment with the auxin transport inhibitor $N$-1-naphthylphthalamic acid (NPA) stimulated root-hair growth in both WT and mpk6 seedlings (Supplementary Fig. S4). Our results indicate that an intact ET signaling pathway is required for mediating the effects of $T$. atroviride on root-hair development, likely involving MPK6.

\section{T. atroviride modifies lateral root formation through a MPK6 and ET-independent mechanism.}

To further investigate whether all the effects of $T$. atroviride on root-system architecture involved components of the ET signaling pathway, we cocultivated WT Arabidopsis seedlings and ET-related mutants with the fungus and primary root growth and lateral root formation were analyzed. When grown under axenic conditions, the primary roots of etrl, ein2, and ein3 mutants were longer than WT. In contrast, when cocultivated with T. atroviride, the growth of the primary roots of WT plants was unaffected, whereas primary root growth of ein 2 and ein3 was inhibited (Fig. 5A). The analysis of lateral root formation showed that the etr1, ein2, and ein 3 mutants already produced three- to sixfold more lateral roots than WT seedlings and manifested a normal induction of lateral roots by T. atroviride (Fig. 5B). Similar effects were observed in the analysis performed with the mpk6 mutant (Fig. 2). These data suggest that, in contrast to root-hair growth, induction of lateral root
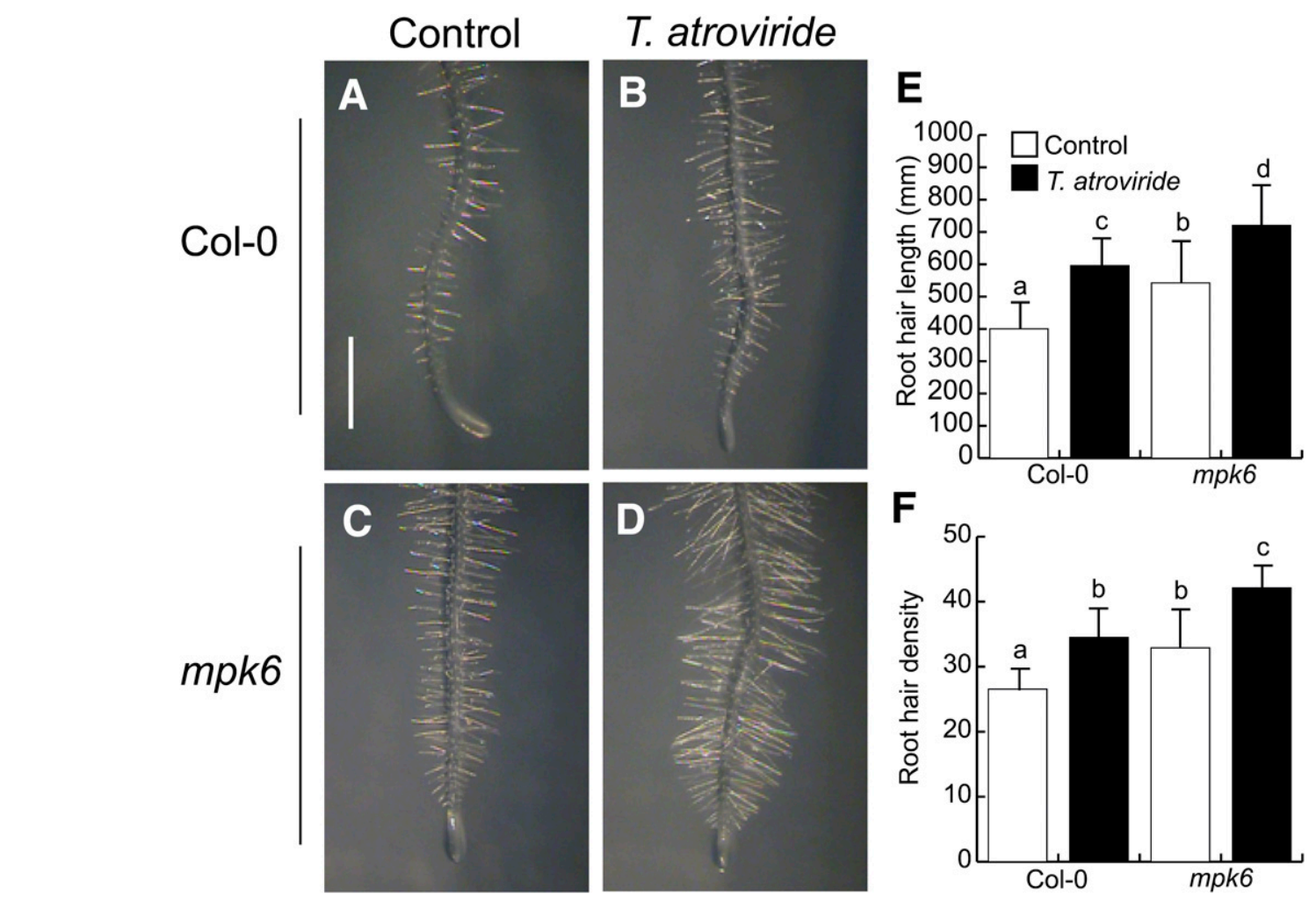

Fig. 1. Trichoderma atroviride effects on root-hair density and development. A to D, Representative roots from wild-type (Col-0) and $m p k 6$ mutant seedlings 6 days after germination, uninoculated (A and C) or inoculated with T. atroviride (B and D). Bar $=500 \mu \mathrm{m}$. E, Average length of root hairs. F, Root-hair density (number of root hairs per cemtimeter). Values shown represent the mean of 100 root hairs \pm standard deviation. Different letters are used to indicate means that differ significantly $(P<0.05)$. 
formation by $T$. atroviride is an MPK6 and ET-independent process.

\section{MPK6 activity is induced in the ctr1 mutant} of Arabidopsis.

The ET signaling pathway is negatively controlled by the master regulator CONSTITUTIVE TRIPLE RESPONSE 1

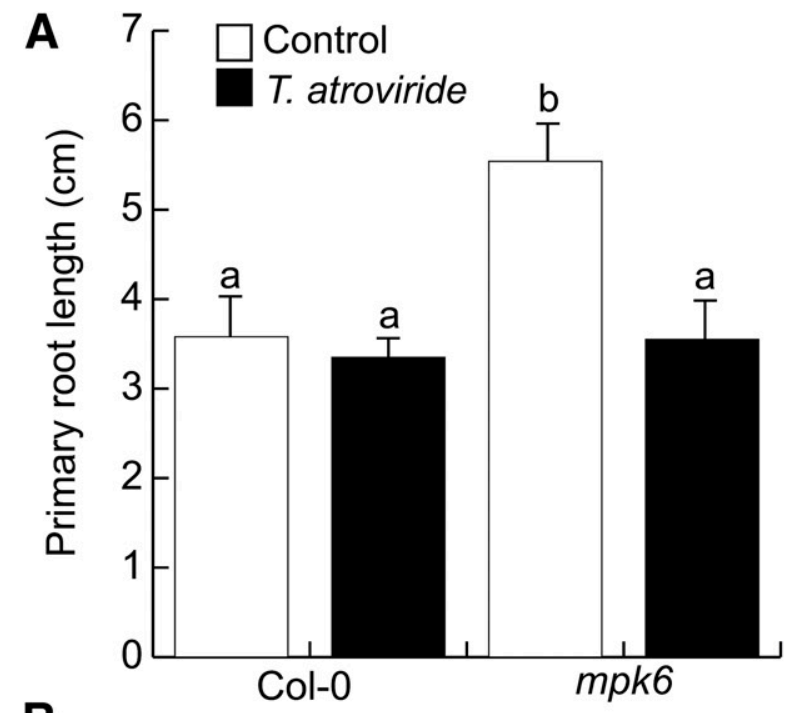

B
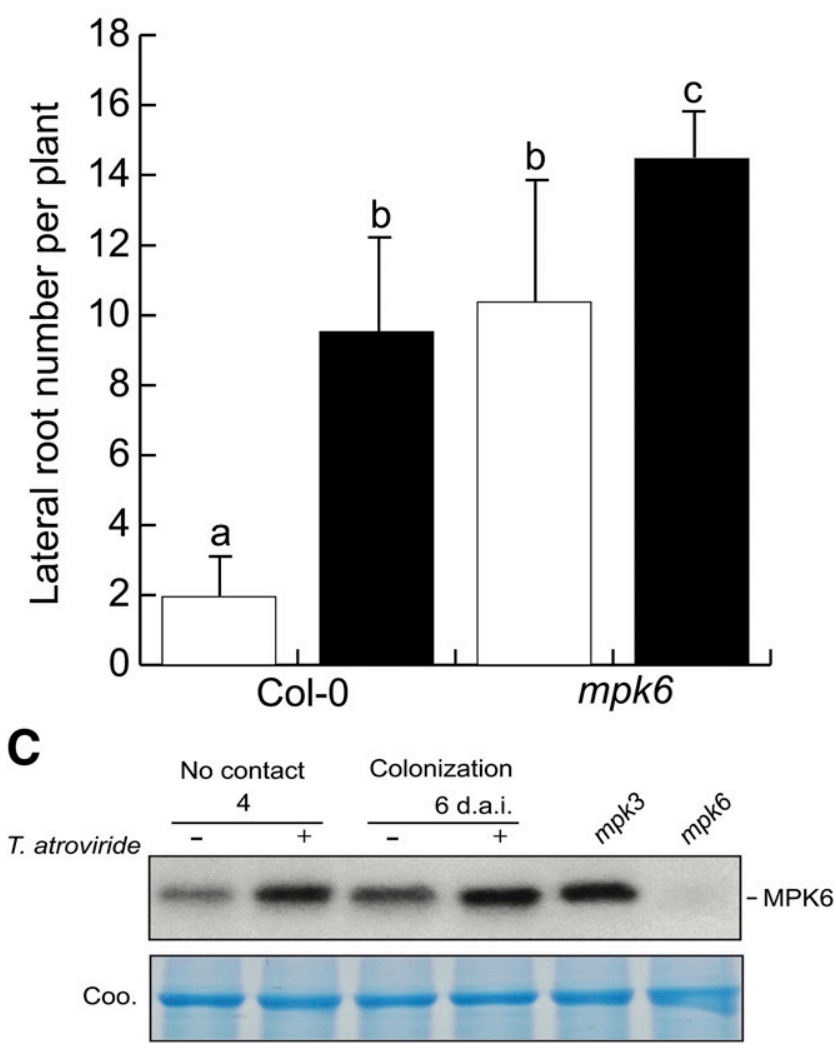

Fig. 2. Trichoderma atroviride effects on root-system architecture and MPK6 activity. A, Primary root length and B, lateral root number of wildtype (Col-0) and mpk6 mutant seedlings 10 days after germination. Root system parameters were quantified for 15 seedlings and different letters are used to indicate means that differ significantly $(P<0.05)$. The experiment was repeated three times with similar results. $\mathbf{C}$, In-gel mitogen-activated protein kinase assays using total protein extracts $(30 \mu \mathrm{g})$ from Col- 0 seedlings growing in the presence of T. atroviride by 4 and 6 days. The Coomassie stained gel (Coo) is shown as loading control and mpk6 and mpk3 mutants were included as reference of MPK6 activity.
(CTR1), which encodes a Raf-like MPKKK (Huang et al. 2003; Kieber et al. 1993). To investigate the contribution of the ET signaling pathway on the MPK6 activity through CTR1, we performed gel kinase assays on the mutant line $c t r l$, growing both under standard light and in darkness conditions. Under a 16-h light and 8-h dark photoperiod, MPK6 activity in the shoots of Col-0 and the ctrl mutant was similar but the kinase activity was increased in the root of the ctrl mutant (Fig. 6A). In the case of shoots and etiolated conditions, no significant differences in MPK6 activity were found between Col-0 and ctrl seedlings. Interestingly, a positive correlation could be established between the increase of MPK6 activity and the repression of the primary root growth in ctrl seedlings (Fig. 6B). In ein2 mutants, a normal or slightly decreased MPK6 activity could be determined (Fig. 6C). These results indicate
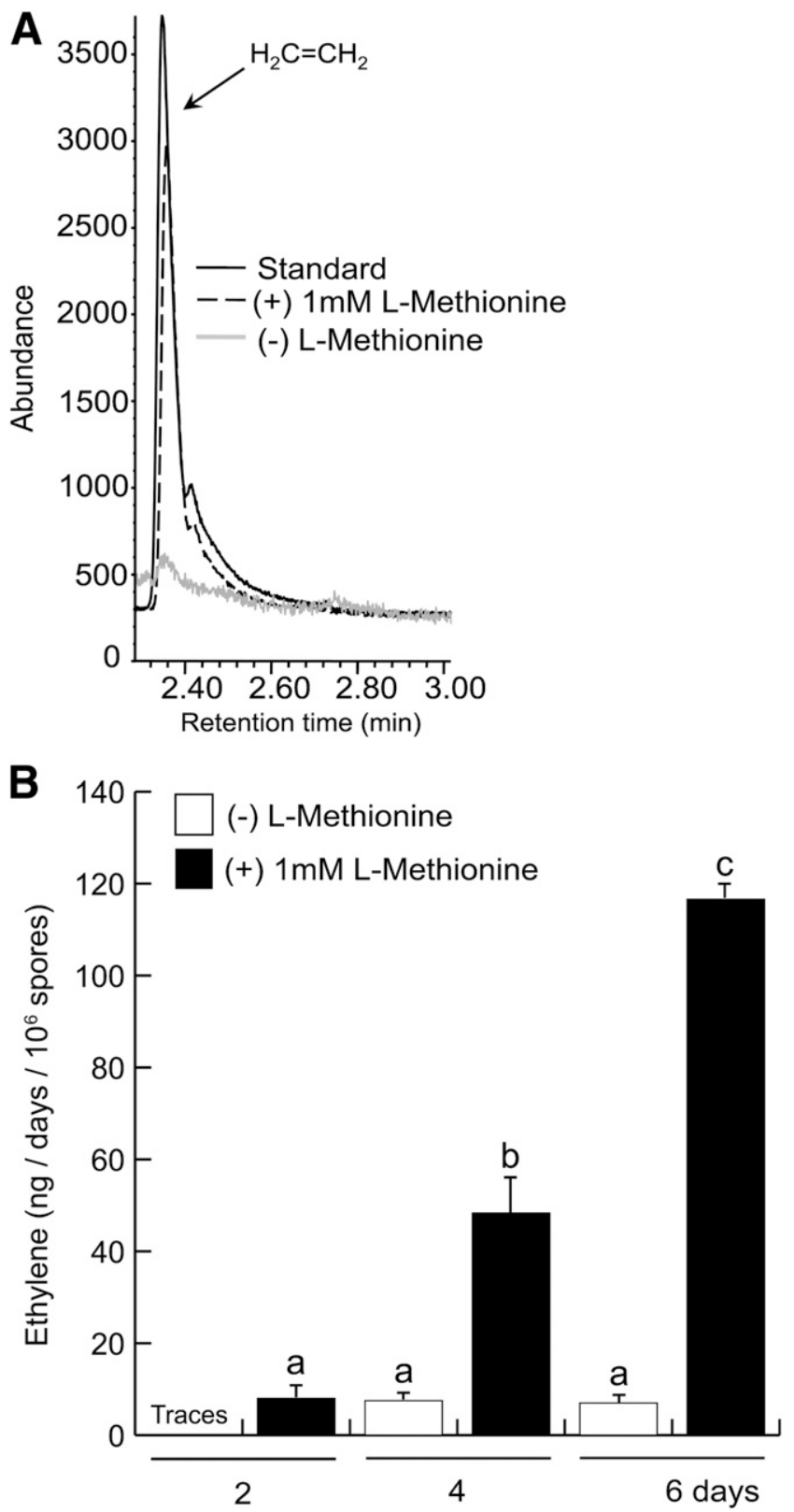

Fig. 3. Trichoderma atroviride ethylene (ET) production. A, ET chromatogram from a gaseous sample obtained of the headspace of a T. atroviride culture medium. B, Production of ET at different intervals of time with or without $1 \mathrm{mM}$ L-methionine. Determinations were from at least four independent samples. Different letters are used to indicate means that differ significantly $(P<0.05)$. 
that MPK6 activity is differentially regulated in shoot and in root systems and that ET signaling influences MPK6 activity particularly in roots.

\section{T. atroviride regulates the activity of MPK6.}

Previously, we identified IAA and auxin-related compounds produced by Trichoderma spp. (Contreras-Cornejo et al. 2009), and we here demonstrate that the indolic compounds accumulate in the growth medium and the fungus also produces ET (Fig. 3). Next, we tested whether $T$. atroviride compounds with auxin activity (IAA, IAAld, and ICAld) affect MPK6 activity. We then evaluated the kinase activity on 10-day-old Arabidopsis seedlings grown in MS $0.2 \times$ medium supplied with different concentrations of these three compounds. The gel kinase assays showed that the MPK6 activity was induced in response to 10 and $100 \mu \mathrm{M}$ IAA, $100 \mu \mathrm{M}$ IAAld, and, slightly, with all concentrations of ICAld assayed (Fig. 7A). Since IAA is the main auxin in plants, we next tested the effect of $1 \mu \mathrm{M}$ IAA on MAPK activity in a short period of time. It was found that MPK6 activity is rapidly activated by IAA (Fig. 7B). These results indicate that auxin-like compounds produced by Trichoderma spp. regulate MPK6 activity.

\section{Expression pattern of the auxin reporter DR5:uidA gene} is altered in the ctr1 mutant.

Our results highlighted that $T$. atroviride alters root-system architecture, possibly via activation of MPK6, which was previously shown to be a negative regulator of primary root growth and lateral root formation (López-Bucio et al. 2014). To investigate a possible ET-auxin crosstalk in this phenomenon, we analyzed the effect of the loss of function of CTR1, a MPKKK considered a master regulator of the ET signaling pathway (Huang et al. 2003; Kieber et al. 1993), on auxin-inducible expression of the DR5:uidA auxin marker (Ulmasov et al. 1997). Then, we generated homozygous F3 ctrl $\times$ DR5:uidA seedlings to compare their $\beta$-glucuronidase (GUS) expression with that of DR5:uidA ( $\mathrm{WT}_{\mathrm{DR} 5}$ ) plants. In $\mathrm{WT}_{\mathrm{DR} 5}$ plants, GUS expression was located in root tips and shoot meristems at 3,6, and 9 days after germination (d.a.g.) (Fig. 8A to F). Interestingly, ctrl/DR5:uidA seedlings of the same age showed short hypocotyls and primary roots, probably correlated with increased accumulation of auxins in the shoot and in the root system (Fig. 8G to L). Particularly, the increased GUS expression in ctr1/DR5:uidA primary roots suggests that both auxin and ET could be related with the prolific growth of root hairs (Fig. 8L). In ctrl/DR5:uidA seedlings cocultivated with T. atroviride, an increased auxin response in root tips was further evidenced (Supplementary Fig. S5). These data show that an ET-auxin crosstalk is involved in the regulation of primary root growth and formation of root hairs in Arabidopsis roots.

\section{DISCUSSION}

In natural environments, plants are continuously exposed to both pathogenic and symbiotic microorganisms that influence their growth and health. Rhizospheric fungi are able to colonize the root system and modulate plant growth and development with implications in crop productivity. Our previous research documented that $T$. virens and $T$. atroviride promote Arabidopsis root growth directly by producing auxin-like compounds (Contreras-Cornejo et al. 2009). However, the molecular
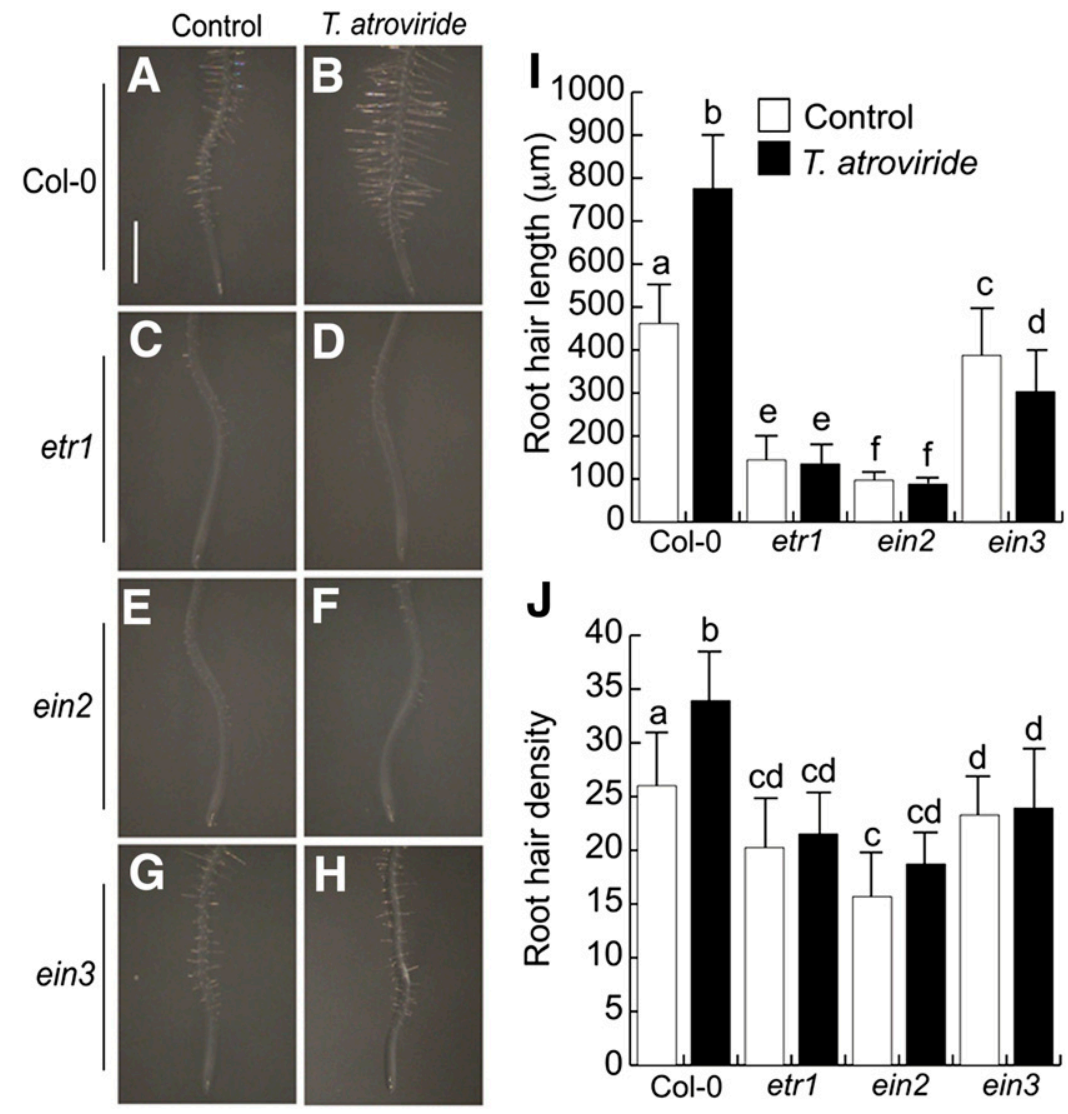

Fig. 4. Trichoderma atroviride effects on root-hair development in ethylene perception mutants. A to $\mathbf{H}$, Representative roots from indicated seedlings grown on Murashige and Skoog $0.2 \times$ medium uninoculated or inoculated with T. atroviride. Bar $=500 \mu \mathrm{m}$. I, Average length of root hairs. J, Root-hair density. Values shown represent the mean of 100 root hairs \pm standard deviation. Different letters are used to indicate means that differ significantly $(P<0.05)$. 
mechanisms that coordinate root responses influenced by these fungi remain to be characterized. There is evidence that external signals from microbes often converge on a set of plant hormones such as auxin and ET, which are responsible for the execution of specific morphogenetic responses in the root (Kazan 2013; Lynch 1972). The large array of responses mediated by these signals is probably achieved by a combinatorial mechanism at the level of biosynthesis, transport, and sensitivity among different tissues (Bennett et al. 2005). MAPKs are classic regulators of most aspects of plant development, including hormone action, cell division, and perception of biotic and abiotic stimuli (Colcombet and Hirt 2008). For instance, plant recognition of microbes leads to rapid activation of MPK3 and MPK6 in Arabidopsis (Bögre et al. 1999; Jonak et al. 1996; Knetsch et al. 1996; Wilson et al. 1997) and, after activation of MPK6, an increase in camalexin levels is detected in Arabidopsis (Ren et al. 2008). In this signaling pathway, a pathogen-inducible transcription factor (WRKY33) is a MPK6 phosphorylation substrate regulating the camalexin biosynthesis (Mao et al. 2011). Our previous and current data show that, through MPK6 activation by T. atroviride, both plant defense responses and root differentiation can be concomitantly regulated. In a previous work, it was reported that Trichoderma spp. induced the accumulation of camalexin (ContrerasCornejo et al. 2011; Velázquez-Robledo et al. 2011). In this
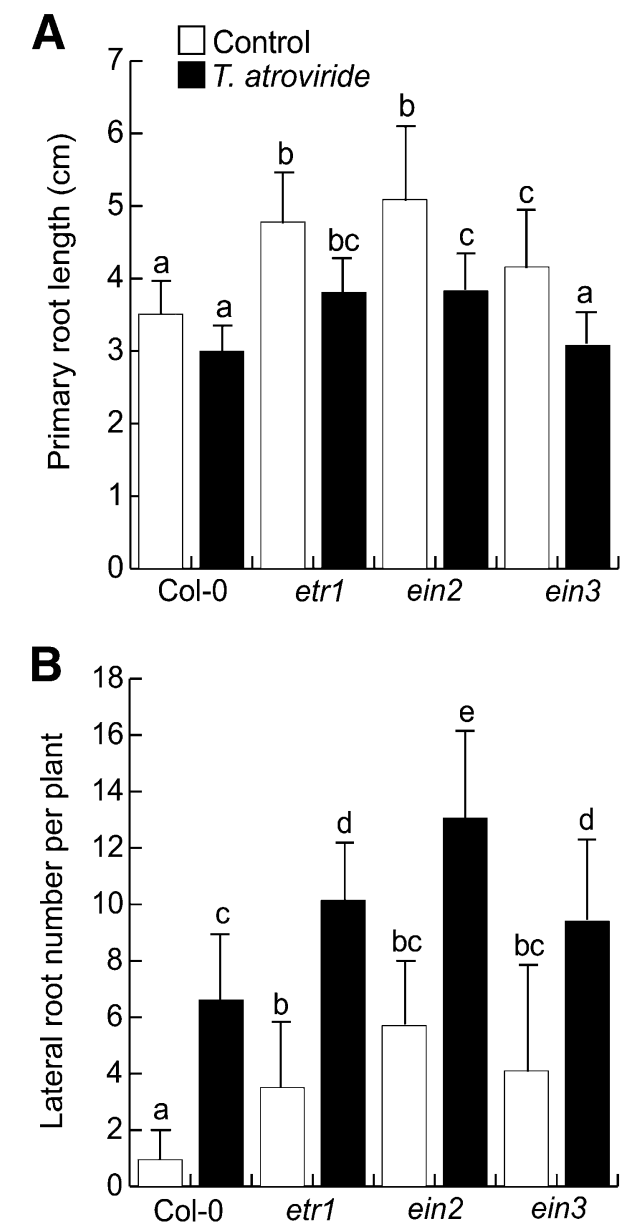

Fig. 5. Trichoderma atroviride effects on the root-system architecture in ethylene perception mutants. A, Primary root length and B, lateral root number of seedlings 10 days after germination. Root-system parameters were quantified for 15 seedlings and different letters are used to indicate means that differ significantly $(P<0.05)$. The experiment was repeated three times with similar results. work, we showed that T. atroviride regulates Arabidopsis root development by the production of ET and auxins involving MPK6 activation, events known to regulate plant responses during plant-microbe interactions (Merkouropoulos et al. 2008; Ren et al. 2008). Recently, it was reported that the MPK6 is a negative regulator of root development, because $m p k 6$ mutants had a longer primary root with more lateral roots and root-hair production than the WT (López-Bucio et al. 2014). Here, our analysis of the previously characterized $m p k 6$ mutant inoculated with $T$. atroviride showed that the number and the length of root hairs increases in a similar fashion in both $m p k 6$ and WT seedlings (Fig. 1), being that increment dependent on ET signaling. Interestingly, the induction of lateral roots in fungus-inoculated mpk6 plants was slightly higher than in WT plants and the primary root growth of the mpk6 mutant was inhibited by inoculation with T. atroviride, whereas the primary

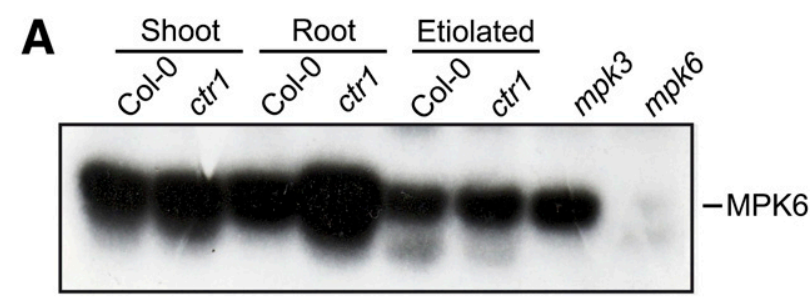

Coo.

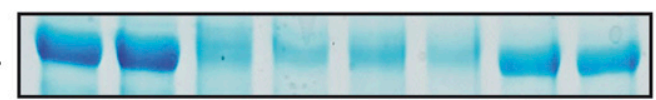

\section{B}

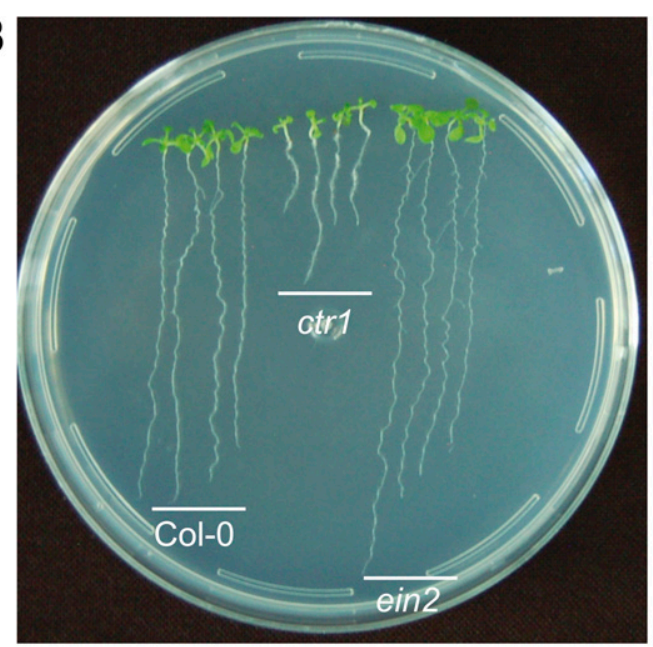

C

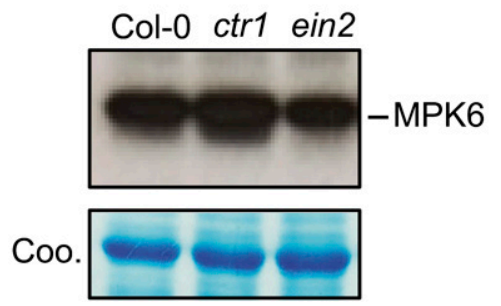

Fig. 6. MPK6 activity in ethylene signaling mutants. A, Mitogen-activated protein kinase activity in organs and etiolated seedlings. Notice that MPK6 activity is increased in the root of the ctrl mutant compared with the wild type (Col-0). B, Root phenotypes of wild type (Col-0), ctr1 and ein2 mutants of 12-day-old seedlings grown in Murashige and Skoog $0.2 \times$ medium. Compared with Col- 0 , ctrl has a shorter primary root and ein2 shows a prolific formation of lateral roots. C, MPK6 activity in Col-0 and the $c t r 1$ and ein 2 mutants. The Coomassie stained gels (Coo) are shown as loading control and $m p k 6$ and $m p k 3$ mutants were included as reference of MPK6 activity. 
roots of WT seedlings were insensitive to this treatment (Fig. 2 ). These observations underscore the complexity of signaling networks controlling the various root morphogenetic events led by $T$. atroviride and support the notion that $T$. atroviride induces changes of the root system likely involving a MAPK signaling pathway.

Root hairs are differentiated epidermal cells directly involved in water and nutrient uptake as well as in interactions with soil microorganisms. As Pitts et al. (1998) reported that ET promoted root-hair elongation in Arabidopsis and we observed that plants inoculated with $T$. atroviride increase the number of root hairs at early stages of the interaction, prior to their physical
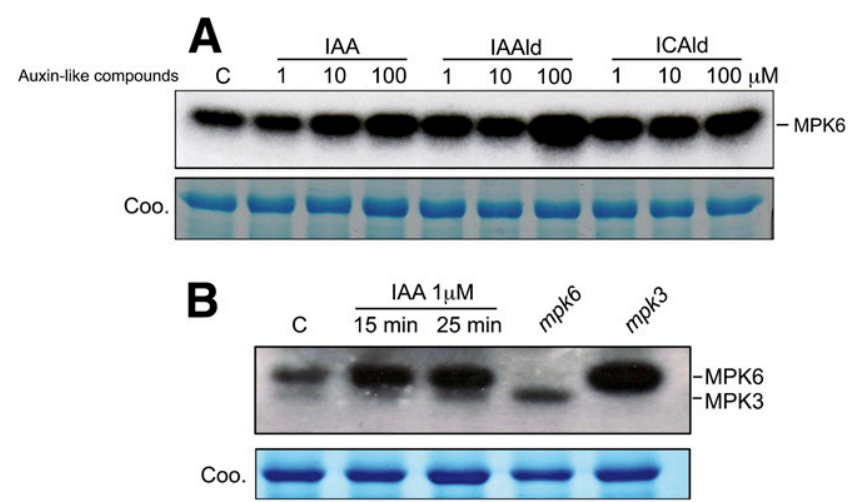

Fig. 7. Compounds with auxin activity produced by Trichoderma atroviride activate MPK6. A, Germination and growth of Arabidopsis seedlings in Murashige and Skoog $0.2 \times$ medium supplied with indole-3-acetic acid (IAA), indole-3-acetaldehyde (IAAld), and indole-3-carboxaldehyde (ICAld) induce MPK6 activity. B, IAA induces a rapid and sustained activation of MPK6. Total protein extracts were prepared from seedlings treated as indicated and $30 \mu \mathrm{g}$ were used for an in-gel mitogen-activate protein kinase assay. The Coomassie stained gels (Coo) are shown as loading control and $m p k 6$ and $m p k 3$ mutants were included as reference of MPK6 activity. contact, we hypothesized that $T$. atroviride could increase the root-hair development by ET production. Then, using gas chromatography-mass spectrometry (GC-mass spectrometry) analysis, we identified T. atroviride ET production in 4- and 6-day fungus cultures. Moreover, the ET production increased when $T$. atroviride culture media was supplemented with L-methionine (Fig. 3), suggesting a canonical biosynthesis pathway. Further, we found that the root-hair induction required an intact ET signaling pathway, as no increased root-hair length or density in response to fungal stimulation was observed on ETsignaling mutants etr1, ein2, and ein3 (Fig. 4) or after treatment with ET inhibitor silver nitrate. Interestingly, the auxin transport inhibitor NPA did not inhibit root-hair development in axenically grown WT seedlings but, rather, stimulated root-hair growth, in contrast to silver nitrate, which inhibits root-hair growth. In response to Trichoderma spp., the combination of NPA with silver nitrate results in root-hair growth inhibition, clearly indicating that ET signaling is important for root-hair responses to Trichoderma spp.

Regarding the effects of $T$. atroviride on lateral root formation and primary root length, we found similar responses in the mpk6 mutant and ET signaling mutants with a slight induction of lateral root production and primary root inhibition caused by fungal cocultivation (Fig. 5). These data indicate that particular root developmental cues are affected by all signals emitted by the fungi and that the net root architectural configuration of plants interacting with Trichoderma spp. likely depends on the timing and degree of root colonization.

It has been reported that auxin and ET act synergistically during root development (Muday et al. 2012). The effect of ET on root growth can be mediated by regulation of auxin biosynthesis or auxin distribution; in turn, elevated levels of auxins enhance ET biosynthesis (Abel et al. 1995; Negi et al. 2008; Yoo et al. 2008). The crosstalk between ET and auxins during fungus-plant interaction is emerging as a key element in signal integration processes. Tuber borchii induces primary root shortening, lateral root, and root-hair production on the host

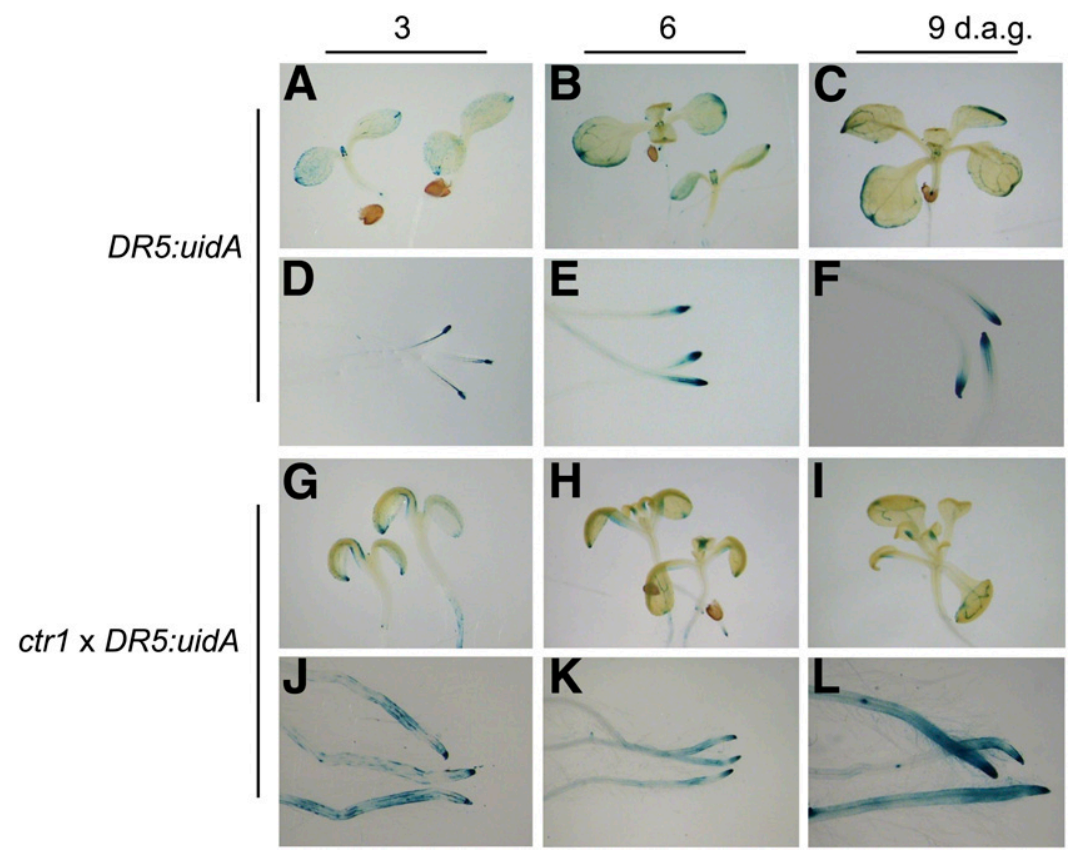

Fig. 8. Ethylene modulates the expression of the DR5 auxin marker gene through CTR1. $\beta$-Glucuronidase (GUS) activity of indicated Arabidopsis seedlings grown for 3, 6, and 9 days after germination (d.a.g.) on Murashige and Skoog 0.2× medium. A to F, Wild-type (DR5:uidA) GUS expression in the shoot and the primary root. G to L, ctrl:DR5:uidA GUS expression in the shoot and the primary root. GUS activity reveals an increased auxin response in the primary root of the $c t r l$ mutant. Representative individuals of at least 10 stained seedlings are shown. The experiment was repeated twice with similar results. 
Cistus incanus and the nonhost Arabidopsis thaliana (Splivallo et al. 2009). The authors demonstrated that the application of a mixture of aminocyclopropane-1-carboxylic acid (ACC) and IAA fully mimicked the root morphology induced by Tuber borchii colony for both host and nonhost plants. Moreover, primary root growth was not inhibited in the Arabidopsis auxin transport mutant aux 1-7 by truffle metabolites, while root branching was less affected in the ET-insensitive mutant ein2-1. Similarly, IAA secreted by Trichoderma strains may promote root growth directly, by stimulating plant cell elongation or cell division, or indirectly, by influencing ET emissions (Viterbo et al. 2010).

MPK6 activity seems to play a central role during T. atroviride root development regulation. It has been demonstrated that MPK6 is activated, supplying the ET precursor ACC to plants. This activation leads to a rapid induction of ET biosynthesis, which is associated with an increase in 1-aminocyclopropane-1-carboxylic acid synthase activity in Arabidopsis (Liu and Zhang 2004). MPK6 also participates in the ET signaling regulating the stability of the transcription factor EIN3 in response to ET (Ouaked et al. 2003; Yoo et al. 2008). Phosphorylation assays in the ctrl mutant suggest that MPK6 could have a key role in the short root phenotype of this mutant, which deserves further attention (Fig. 6). We also found that the compounds with auxin activity released by Trichoderma spp. induce MPK6 activity (Fig. 7). Then, it is tempting to speculate that MPK6 could be acting as an integrator of auxin and ET signals. This speculation is supported by an auxininducible gene expression analysis on homozygous $c t r 1 / D R 5$ : uidA seedlings, which clearly shows an increased auxininducible expression independent of fungal colonization (Fig. 8). According to these data, we propose a model in which T. atroviride induces MPK6 accompanied by ET and auxin production (Fig. 9). T. atroviride affects root morphogenetic programs, apparently inducing the MPK6 activation through ET and indolic compound biosynthesis. ET and auxins induce root-hair production in an ET-dependent pathway, whereas ET could regulate the auxin biosynthesis and distribution to enhance lateral root production. These two hormones can act synergistically to inhibit primary root growth by an overactivation of MPK6, which acts as a negative regulator of primary root growth and lateral root formation (Fig. 9). In consequence, MPK6 and its MAPK associated cascade, likely including CTR1, seems to be a regulation node to one or both maintain or amplify the hormonal effects underlying plant development or defense or both. To our knowledge, the short-root phenotype of ctrl mutants has not been previously reported. Apparently, ctrl primary roots enter a determinate developmental pathway caused by quiescent center-cell differentiation and halted cell division in primary root meristems (A. Méndez-Bravo and J. López-Bucio, personal communication). We cannot exclude the possibility that other molecules, such as small peptides, volatiles, or diffusibles, may also affect root development through MPK6 signaling. Our work provides conclusive data demonstrating that a MAPK module including MPK6 acts as a signal transducer during root development mediated by Trichoderma spp. In this scenario, the induction of MPK6 activity by $T$. atroviride auxin-like compounds could enhance ET biosynthesis, thus regulating the root-hair development in an ET-dependent pathway. In turn, the enhanced ET production could influence auxin biosynthesis or distribution impacting the initiation of lateral roots, all together suggesting a positive feedback between auxins and ET during a beneficial fungal-plant interaction.

\section{MATERIALS AND METHODS}

\section{Plant material and growth conditions.}

Arabidopsis thaliana ecotype Columbia-0 (Col-0) and several mutant lines were used in this work. Lines $m p k 6-2$ (Liu and Zhang 2004) and $m p k 3-1$ (Wang et al. 2007) are mutants affected in protein kinases; etrl-3 (Hua and Meyerowitz 1998), ein2-1 (Guzmán and Ecker 1990), ein3-1 (Chao et al. 1997), and ctrl-1 (Kieber et al. 1993) are ET-related mutants. DR5: uidA (Ulmasov et al. 1997) is an auxin-responsive marker. A homozygous ctr1/DR5:uidA line used to analyze auxin signaling in the root system as affected by the ET pathway was generated in this work. All the seeds were surface-sterilized, and were then germinated and grown on agar plates containing $0.2 \times$ MS medium (MS basal salts mixture; Sigma), basal or supplied with the indicated compounds. Plates were placed vertically at a 65-degree angle to allow root growth along the agar surface and unimpeded aerial growth of the hypocotyls. Plants were grown at $24^{\circ} \mathrm{C}$ in a chamber with a 16 -h light $\left(200 \mu \mathrm{mol} \mathrm{m} \mathrm{m}^{2} \mathrm{~s}^{-1}\right), 8$-h dark photoperiod.

\section{Fungal growth and plant inoculation experiments.}

Trichoderma atroviride IMI 206040 used in this was previously evaluated in vitro for their ability to promote plant growth in Arabidopsis. Fungal spore densities of $10^{6}$ were inoculated placing the spores at $5 \mathrm{~cm}$ in the opposite ends of agar plates containing 4-d.a.g. Arabidopsis seedlings (10 seedlings per plate). Plates were arranged in a completely randomized design. The seedlings were cultivated in a Percival AR95L growth chamber at the temperature and photoperiod conditions already indicated.

\section{Plant growth measurements.}

At 4 d.a.i., total fresh weight was determined on an analytical scale for three groups of 10 plants. Growth of primary roots was registered using a ruler. Lateral root numbers were determined by counting the lateral roots present in the primary root. To analyze the effect of $T$. atroviride on root cell differentiation, the primary root zone was photographed 2 days after inoculation, using a stereoscopic microscope (Leica MZ6; Leica Microsystems). Measurements were determined using Image J Tool software; pixel areas were calibrated based on a known distance. Root hairs were measured in a region of $500 \mu \mathrm{m}$ at

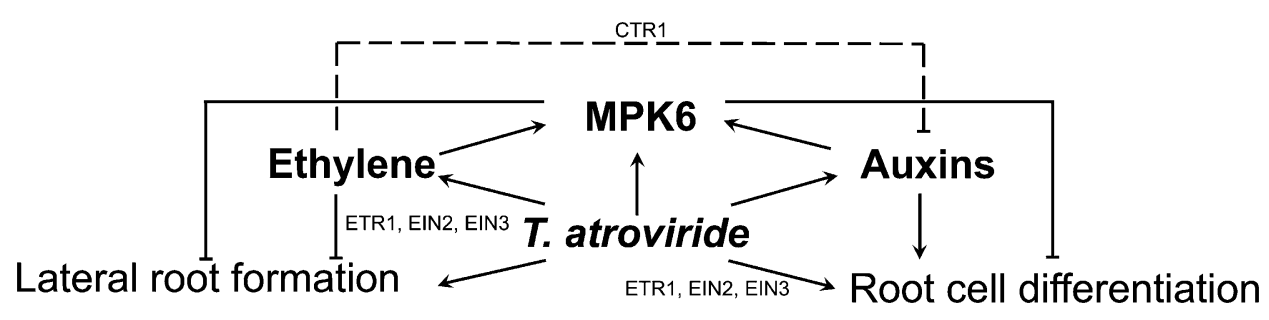

Fig. 9. Proposed model for the signaling pathways controlled by Trichoderma atroviride in Arabidopsis for modulating the root-system architecture. T. atroviride induces the activation of plant MPK6 that translates fungal signals into cellular responses. Plant growth regulators, such as auxins and ethylene, produced by the fungus control several aspects of the root growth but, simultaneously, can modulate the MPK6 activity, which also affects root development. 
approximately $1 \mathrm{~cm}$ from the primary root tip. The average length of root hairs was determined measuring 100 hairs for each root, taking as a reference the root protoxylematic plane to locate the radical hair base in the epidermal cell.

\section{Indolic compound determinations.}

Arabidopsis WT seedlings (Col-0) were grown and inoculated as described above. IAA and indolic compound determinations were done by GC-mass spectrometry from the MS agar portion of the petri dishes after 6 days of fungal growth. Seedlings and mycelia were removed from the agar surface. A quantity of 20 to $28 \mathrm{~g}$ of MS agar was removed from each plate with a spatula, was placed in a flask with $100 \mathrm{ml}$ of deionized water, and was homogenized and filtered. To recover IAA, samples were acidified to $\mathrm{pH} 3$ with concentrated $\mathrm{HCl}$. IAAld and ICAld were obtained when the aqueous extracts from the agar were adjusted to $\mathrm{pH} 7$, using $10 \% \mathrm{KOH}$. Aqueous solutions were mixed with $100 \mathrm{ml}$ of ethyl acetate and were vortexed for $1 \mathrm{~min}$. The upper phase was concentrated to dryness at $55^{\circ} \mathrm{C}$, was redissolved in $3.0 \mathrm{ml}$ of ethyl acetate, was trasferred to vials, and was evaporated to dryness under a stream on nitrogen. Samples containing IAAld and ICAld were redissolved in $50 \mu \mathrm{l}$ of ethyl acetate. For IAA determinations, IAA was methyl esterified with $500 \mu \mathrm{l}$ of acetyl chloride in $2 \mathrm{ml}$ of dry methanol, was sonicated for $15 \mathrm{~min}$, and was heated at $75^{\circ} \mathrm{C}$ for $1 \mathrm{~h}$. The IAA methyl ester was evaporated under a stream of nitrogen and redissolved in $50 \mu \mathrm{l}$ of ethyl acetate.

The indolic compounds were analyzed in an Agilent 6850 Series II gas chromatograph equipped with an Agilent MS detector model 5973 and a $30 \mathrm{~m} \times 0.2 \mu \mathrm{m} \times 0.25 \mathrm{~mm}, 5 \%$ phenyl methyl silicone capillary column (HP-5 MS). Operating conditions used helium at a rate of $1 \mathrm{ml} \mathrm{min}^{-1}$ as carrier gas, $300^{\circ} \mathrm{C}$ detector temperature, and $250^{\circ} \mathrm{C}$ injector temperature. The volume of the injected sample was $1 \mu \mathrm{l}$. The column was held for $5 \mathrm{~min}$ at $150^{\circ} \mathrm{C}$ and was programmed at $5^{\circ} \mathrm{C}$ per minute to a final temperature of $278^{\circ} \mathrm{C}$ for $5 \mathrm{~min}$. Indolic compounds were identified by comparison with a mass spectra library (National Institute of Standards and Technology, Environmental Protection Agency, National Institutes of Health; Chem Station; Hewlett-Packard). The identities of the indolic compounds were further confirmed by comparison of the retention time $(\mathrm{Rt})$ in the fungal extract with samples of the pure IAAld $(\mathrm{Rt}=8.88 \mathrm{~min})$, ICAld $(\mathrm{Rt}=10.98 \mathrm{~min})$, and IAA $(\mathrm{Rt}=$ $11.02 \mathrm{~min}$ ) standards (Sigma). A selected ion-monitoring analysis was used to verify the presence of these indolic compounds in the samples. The molecular ions were monitored after electron impact ionization $(70 \mathrm{eV})$. For IAAld, mass-tocharge ratios $(\mathrm{m} / \mathrm{z})$ were $\mathrm{m} / \mathrm{z}, 130$ and $159\left[\mathrm{M}^{+}\right]$; for ICAld, they were $\mathrm{m} / z, 116$ and $145\left[\mathrm{M}^{+}\right]$; and for IAA methyl ester, they were $\mathrm{m} / z, 130$ and $189\left[\mathrm{M}^{+}\right]$. To estimate the amount of compounds produced by $T$. atroviride, we constructed individual calibration curves for each compound.

\section{ET collection and analysis.}

T. atroviride was inoculated on petri dishes containing MS $0.2 \times$ medium for 2,4 , and 6 days days, for measuring the accumulation of ET in the head-space. Gaseous hormone was collected using a gas-tight (Batch H08-A9817) syringe. The identification and determination of ET were performed using an Agilent 6850 Series II gas chromatograph equipped with a 30-m MS Q-Plot column GC-mass spectrometry system. GCselected ion monitoring mass spectrometry and retention time were established for ET $(\mathrm{m} / \mathrm{z}, 26$ and 27, $2.39 \mathrm{~min})$. The operating conditions used were: $1 \mathrm{ml} \mathrm{min}{ }^{-1}$ of helium as carrier gas, $250^{\circ} \mathrm{C}$ detector temperature, and $110^{\circ} \mathrm{C}$ injector temperature. The column was held for $4 \mathrm{~min}$ at $60^{\circ} \mathrm{C}$ and the samples were run at isotermic conditions in the splitless mode. To estimate the amount of ET, columns were injected with $200 \mu$ of gas taken from $2 \mathrm{ml}$ of sample treated previously with $2 \mathrm{ml}$ of $\mathrm{KOH}$ $1 \mathrm{~N}$ to eliminate the $\mathrm{CO}_{2}$ produced by the fungus that could interfere with the peak of the ET. Additionally, we constructed a curve with a pure standard (Sigma).

\section{Protein extraction.}

Frozen seedlings were ground in liquid nitrogen and were thawed in extraction buffer (100 mM HEPES [pH 7.5], $5 \mathrm{mM}$ EDTA, $5 \mathrm{mM}$ EGTA, $10 \mathrm{mM}$ dithiothreitol [DTT], $10 \mathrm{mM}$ $\mathrm{Na}_{3} \mathrm{VO} 4,10 \mathrm{mM} \mathrm{NaF}, 50 \mathrm{mM} \beta$-glycerophosphate, $10 \%$ glycerol, $7.5 \%$ polyvinylpolypyrrolidone), supplied with a protease inhibitor mixture (as recommended by Sigma). The ground slurry was centrifuged at $20,000 \times g$ for $20 \mathrm{~min}$ at $4^{\circ} \mathrm{C}$. Supernatants were collected into new tubes, were frozen in liquid nitrogen, and were stored at $-80^{\circ} \mathrm{C}$ until their use. The protein concentration was determined using the Bio-Rad protein assay kit with bovine serum albumin (BSA) as standard.

\section{In-gel kinase assays.}

The in-gel kinase assay was performed as described by $\mathrm{Lu}$ et al. (2002). Briefly, 50- $\mu$ g aliquots of total protein extracted from plant tissue were fractionated on a sodium dodecyl sulfate $10 \%$ polyacrylamide gel containing $0.25 \mathrm{mg}$ myelin basic protein per milliliter (Sigma) as substrate for the MAPKs. After electrophoresis, the gel was washed three times for $30 \mathrm{~min}$ each with $25 \mathrm{mM}$ Tris, pH 7.5, $0.5 \mathrm{mM}$ DTT, $0.1 \mathrm{mM} \mathrm{Na}_{3} \mathrm{VO}_{4}, 5 \mathrm{mM}$ $\mathrm{NaF}, 0.5 \mathrm{mg}$ of BSA per milliliter, and $0.1 \%$ (vol/vol) Triton $\mathrm{X}-100$, at room temperature. Proteins in the gel were renatured by incubating the gel in $25 \mathrm{mM}$ Tris, $\mathrm{pH} 7.5,1 \mathrm{mM}$ DTT, $0.1 \mathrm{mM}$ $\mathrm{Na}_{3} \mathrm{VO}_{4}$, and $5 \mathrm{mM} \mathrm{NaF}$ at $4^{\circ} \mathrm{C}$ overnight with three changes of buffer. The kinase reactions were carried out by incubating the gel in $30 \mathrm{ml}$ of reaction buffer $(25 \mathrm{mM}$ Tris, $\mathrm{pH} 7.5,2 \mathrm{mM}$ EGTA, $12 \mathrm{mM} \mathrm{MgCl}_{2}, 1 \mathrm{mM}$ DTT, $0.1 \mathrm{mM} \mathrm{Na}_{3} \mathrm{VO}_{4}, 200 \mathrm{nM}$ ATP, and $50 \mu \mathrm{Ci}$ of $\left[\gamma-{ }^{32} \mathrm{P}\right]$ ATP with $>4,000 \mathrm{Ci} / \mathrm{mmol} ; 1 \mathrm{Ci}=37$ $\mathrm{GBq}$ ) for $60 \mathrm{~min}$ at room temperature. To remove free ${ }^{32} \mathrm{P}$, the gel was repeatedly washed at room temperature with several changes of $5 \%(\mathrm{wt} / \mathrm{vol})$ trichloroacetic acid and $1 \%(\mathrm{wt} / \mathrm{vol}) \mathrm{NaPP}_{\mathrm{i}}$ until ${ }^{32} \mathrm{P}$ radioactivity in the wash solution was barely detectable. Then, the gel was dried under vacuum on Whatman 3MM paper and was used to expose an X-ray film. Protein sizes were estimated by using molecular mass markers (Bio-Rad).

\section{Data analysis.}

Experiments were statistically analyzed in the SPSS 10 program (SPSS, Chicago). Multivariate analyses with a Tukey's post hoc test were used for testing differences in the root system architecure. Different letters were used to indicate means that differed significantly $(P \leq 0.05)$.

\section{ACKNOWLEDGMENTS}

We thank J. Altamirano for his support and technical improvement for ET determination. We are thankful to P. Guzmán and T. Guilfoyle for kindly providing us with Arabidopsis mutant seeds. H. A. Contreras-Cornejo is indebted to CONACYT for a doctoral fellowship. This work was supported by grants from the Consejo Nacional de Ciencia y Tecnología (CONACYT, México, grant number 43978), the Consejo de la Investigación Científica (UMSNH, México, grant number CIC 2.26), and the UNAM-DGAPAPAPIIT (grant IN207014 to Á. A. Guevara-García, J. S. López-Bucio, and M. Ramos-Vega)

\section{LITERATURE CITED}

Abel, S., Nguyen, M. D., Chow, W., and Theologis, A. 1995. ACS4, a primary indoleacetic acid-responsive gene encoding 1-aminocyclopropane1-carboxylate synthase in Arabidopsis thaliana. Structural characterization, expression in Escherichia coli, and expression characteristics in response to auxin [corrected]. J. Biol. Chem. 270:19093-19099. 
Acharya, B. R., and Assmann, S. M. 2009. Hormone interactions in stomatal function. Plant Mol. Biol. 69:451-462.

Barbieri, P., and Galli, E. 1993. Effect on wheat root development of inoculation with an Azospirillum brasilense mutant with altered indole3-acetic acid production. Res. Microbiol. 144:69-75.

Bennett, M., Bellini, C., and Van Der Straeten, D. 2005. Integrative biology: Dissecting cross-talk between plant signaling pathways. Physiol. Plant. 123:109.

Bleecker, A. B., and Kende, H. 2000. Ethylene: A gaseous signal molecule in plants. Annu. Rev. Cell Dev. Biol. 16:1-18.

Bögre, L., Calderini, O., Binarova, P., Mattauch, M., Till, S., Kiegerl, S., Jonak, C., Pollaschek, C., Barker, P., Huskisson, N. S., Hirt, H., and Heberle-Bors, E. 1999. A MAP kinase is activated late in plant mitosis and becomes localized to the plane of cell division. Plant Cell 11:101-113.

Brader, G., Djamei, A., Teige, M., Palva, E. T., and Hirt, H. 2007. The MAP kinase kinase MKK2 affects disease resistance in Arabidopsis. Mol. Plant-Microbe Interact. 20:589-596.

Chao, Q., Rothenberg, M., Solano, R., Roman, G., Terzaghi, W., and Ecker, J. R. 1997. Activation of the ethylene gas response pathway in Arabidopsis by the nuclear protein ETHYLENE-INSENSITIVE3 and related proteins. Cell 89:1133-1144.

Colcombet, J., and Hirt, H. 2008. Arabidopsis MAPKs: A complex signalling network involved in multiple biological processes. Biochem. J. 413:217-226.

Contreras-Cornejo, H. A., Macías-Rodríguez, L., Cortés-Penagos, C., and López-Bucio, J. 2009. Trichoderma virens, a plant beneficial fungus, enhances biomass production and promotes lateral root growth through an auxin-dependent mechanism in Arabidopsis. Plant Physiol. 149: 1579-1592.

Contreras-Cornejo, H. A., Macías-Rodríguez, L., Beltrán-Peña, E., HerreraEstrella, A., and López-Bucio, J. 2011. Trichoderma-induced plant immunity likely involves both hormonal- and camalexin-dependent mechanisms in Arabidopsis thaliana and confers resistance against necrotrophic fungi Botrytis cinerea. Plant Signal. Behav. 6:1554-1563.

Contreras-Cornejo, H. A., Ortiz-Castro, R., and López-Bucio, J. 2013. Promotion of plant growth and the induction of systemic defence by Trichoderma: Physiology, genetics and gene expression. Pages 175-196 in: Trichoderma Biology and Applications. P. K. Mukherjee,ed. CAB International, London.

Contreras-Cornejo, H. A., Macías-Rodríguez, L., Alfaro-Cuevas, R., and López-Bucio, J. 2014. Trichoderma spp. improve growth of Arabidopsis seedlings under salt stress through enhanced root development, osmolite production, and $\mathrm{Na}^{+}$elimination through root exudates. Mol. Plant-Microbe Interact. 27:503-514.

Dharmasiri, N., Dharmasiri, S., Weijers, D., Lechner, E., Yamada, M., Hobbie, L., Ehrismann, J. S., Jürgens, G., and Estelle, M. 2005. Plant development is regulated by a family of auxin receptor $\mathrm{F}$ box proteins. Dev. Cell 9:109-119.

Guzmán, P., and Ecker, J. R. 1990. Exploiting the triple response of Arabidopsis to identify ethylene-related mutants. Plant Cell 2:513-523.

Hua, J., and Meyerowitz, E. M. 1998. Ethylene responses are negatively regulated by a receptor gene family in Arabidopsis thaliana. Cell 94: 261-271.

Huang, Y., Li, H., Hutchison, C. E., Laskey, J., and Kieber, J. J. 2003 Biochemical and functional analysis of CTR1, a protein kinase that negatively regulates ethylene signaling in Arabidopsis. Plant J. 33:221-233.

Johnson, P. R., and Ecker, J. R. 1998. The ethylene gas signal transduction pathway: A molecular perspective. Annu. Rev. Genet. 32:227-254.

Jonak, C., Kiegerl, S., Ligterink, W., Barker, P. J., Huskisson, N. S., and Hirt, H. 1996. Stress signaling in plants: A mitogen-activated protein kinase pathway is activated by cold and drought. Proc. Natl. Acad. Sci. U.S.A. 93:11274-11279.

Kazan, K. 2013. Auxin and the integration of environmental signals into plant root development. Ann. Bot. (Lond.) 112:1655-1665.

Khatabi, B., Molitor, A., Lindermayr, C., Pfiffi, S., Durner, J., von Wettstein, D., Kogel, K. H., and Schäfer, P. 2012. Ethylene supports colonization of plant roots by the mutualistic fungus Piriformospora indica. PLoS ONE 7:e35502.

Kieber, J. J., Rothenberg, M., Roman, G., Feldmann, K. A., and Ecker, J. R. 1993. CTR1, a negative regulator of the ethylene response pathway in Arabidopsis, encodes a member of the raf family of protein kinases. Cell 72:427-441.

Knetsch, M., Wang, M., Snaar-Jagalska, B. E., and Heimovaara-Dijkstra, S. 1996. Abscisic acid induces mitogen-activated protein kinase activation in barley aleurone protoplasts. Plant Cell 8:1061-1067.

Liu, Y., and Zhang, S. 2004. Phosphorylation of 1-aminocyclopropane-1carboxylic acid synthase by MPK6, a stress-responsive mitogenactivated protein kinase, induces ethylene biosynthesis in Arabidopsis. Plant Cell 16:3386-3399.
López-Bucio, J. S., Dubrovsky, J. G., Raya-González, J., UgartecheaChirino, Y., López-Bucio, J., de Luna-Valdez, L. A., Ramos-Vega, M., León, P., and Guevara-García, A. A. 2014. Arabidopsis thaliana mitogen-activated protein kinase 6 is involved in seed formation and modulation of primary and lateral root development. J. Exp. Bot. 65 169-183.

Lu, C., Han, M. H., Guevara-Garcia, A., and Fedoroff, N. V. 2002. Mitogenactivated protein kinase signaling in postgermination arrest of development by abscisic acid. Proc. Natl. Acad. Sci. U.S.A. 99:15812-15817.

Lynch, J. M. 1972. Identification of substrates and isolation of microorganisms responsible for ethylene production in the soil. Nature 240:45-46.

Malhotra, M., and Srivastava, S. 2008. An ipdC gene knock-out of Azospirillum brasilense strain SM and its implications on indole-3-acetic acid biosynthesis and plant growth promotion. Antonie van Leeuwenhoek 93:425-433.

Mao, G., Meng, X., Liu, Y., Zheng, Z., Chen, Z., and Zhang, S. 2011. Phosphorylation of a WRKY transcription factor by two pathogenresponsive MAPKs drives phytoalexin biosynthesis in Arabidopsis. Plant Cell 23:1639-1653.

Merkouropoulos, G., Andreasson, E., Hess, D., Boller, T., and Peck, S. C. 2008. An Arabidopsis protein phosphorylated in response to microbial elicitation, AtPHOS32, is a substrate of MAP kinases 3 and 6. J. Biol Chem. 283:10493-10499.

Muday, G. K., Rahman, A., and Binder, B. M. 2012. Auxin and ethylene: Collaborators or competitors? Trends Plant Sci. 17:181-195.

Negi, S., Ivanchenko, M. G., and Muday, G. K. 2008. Ethylene regulates lateral root formation and auxin transport in Arabidopsis thaliana. Plant J. 55:175-187.

Ouaked, F., Rozhon, W., Lecourieux, D., and Hirt, H. 2003. A MAPK pathway mediates ethylene signaling in plants. EMBO (Eur. Mol. Biol. Organ.) J. 22:1282-1288.

Patten, C. L., and Glick, B. R. 2002. Role of Pseudomonas putida indoleacetic acid in development of the host plant root system. Appl. Environ. Microbiol. 68:3795-3801.

Pitts, R. J., Cernac, A., and Estelle, M. 1998. Auxin and ethylene promote root hair elongation in Arabidopsis. Plant J. 16:553-560.

Ren, D., Liu, Y., Yang, K. Y., Han, L., Mao, G., Glazebrook, J., and Zhang, S. 2008. A fungal-responsive MAPK cascade regulates phytoalexin biosynthesis in Arabidopsis. Proc. Natl. Acad. Sci. U.S.A. 105: 5638-5643.

Růzicka, K., Ljung, K., Vanneste, S., Podhorská, R., Beeckman, T., Friml, J., and Benková, E. 2007. Ethylene regulates root growth through effects on auxin biosynthesis and transport-dependent auxin distribution. Plant Cell 19:2197-2212.

Sánchez-Rodríguez, C., Rubio-Somoza, I., Sibout, R., and Persson, S. 2010. Phytohormones and the cell wall in Arabidopsis during seedling growth. Trends Plant Sci. 15:291-301.

Splivallo, R., Fischer, U., Göbel, C., Feussner, I., and Karlovsky, P. 2009. Truffles regulate plant root morphogenesis via the production of auxin and ethylene. Plant Physiol. 150:2018-2029.

Suzuki, S., He, Y., and Oyaizu, H. 2003. Indole-3-Acetic acid production in Pseudomonas fluorescens HP72 and its association with suppression of creeping bentgrass brown patch. Curr. Microbiol. 47:138-143.

Ulmasov, T., Murfett, J., Hagen, G., and Guilfoyle, T. J. 1997. Aux/IAA proteins repress expression of reporter genes containing natural and highly active synthetic auxin response elements. Plant Cell 9:1963-1971.

Velázquez-Robledo, R., Contreras-Cornejo, H. A., Macías-Rodriguez, L., Hernández-Morales, A., Aguirre, J., Casas-Flores, S., López-Bucio, J. and Herrera-Estrella, A. 2011. Role of the 4-phosphopantetheinyl transferase of Trichoderma virens in secondary metabolism and induction of plant defense responses. Mol. Plant-Microbe Interact. 24:1459-1471.

Viterbo, A., Landau, U., Kim, S., Chernin, L., and Chet, I. 2010. Characterization of ACC deaminase from the biocontrol and plant growth-promoting agent Trichoderma asperellum T203. FEMS Microbiol. Lett. 305:42-48.

Wang, H., Ngwenyama, N., Liu, Y., Walker, J. C., and Zhang, S. 2007. Stomatal development and patterning are regulated by environmentally responsive mitogen-activated protein kinases in Arabidopsis. Plant Cell 19:63-73

Wilson, C., Voronin, V., Touraev, A., Vicente, O., and Heberle-Bors, E. 1997. A developmentally regulated MAP kinase activated by hydration in tobacco pollen. Plant Cell 9:2093-2100.

Yoo, S. D., Cho, Y. H., Tena, G., Xiong, Y., and Sheen, J. 2008. Dual control of nuclear EIN3 by bifurcate MAPK cascades in $\mathrm{C}_{2} \mathrm{H}_{4}$ signalling. Nature 451:789-795.

Zhang, T., Liu, Y., Yang, T., Zhang, L., Xu, S., Xue, L., and An, L. 2006. Diverse signals converge at MAPK cascades in plant. Plant Physiol Biochem. 44:274-283. 